\title{
Performance of the NASA Beacon Receiver for the Alphasat Aldo Paraboni TDP5 Propagation Experiment
}

\author{
James Nessel, Jacquelynne Morse, \\ Michael Zemba \\ NASA Glenn Research Center 4800 \\ 21000 Brookpark Rd. MS 54-1 \\ Cleveland, OH 44135 \\ +1 216-433-2546 \\ james.a.nessel@nasa.gov
}

\author{
Carlo Riva, Lorenzo Luini \\ Politecnico di Milano \\ Piazza Leonardo da Vinci, 32 \\ 20133 Milano, Italy \\ +39 02-23993659 \\ carlo.riva@polimi.it
}

\begin{abstract}
NASA Glenn Research Center (GRC) and the Politecnico di Milano (POLIMI) have initiated a joint propagation campaign within the framework of the Alphasat propagation experiment to characterize rain attenuation, scintillation, and gaseous absorption effects of the atmosphere in the $40 \mathrm{GHz}$ band. NASA GRC has developed and installed a $\mathrm{K} / \mathrm{Q}$-band (20/40 GHz) beacon receiver at the POLIMI campus in Milan, Italy, which receives the $20 / 40 \mathrm{GHz}$ signals broadcast from the Alphasat Aldo Paraboni Technology Demonstration Payload (TDP) \#5 beacon payload. The primary goal of these measurements is to develop a physical model to improve predictions of communications systems performance within the Q-band. Herein, we describe the design and preliminary performance of the NASA propagation terminal, which has been installed and operating in Milan since June 2014. The receiver is based upon a validated Fast Fourier Transform (FFT) I/Q digital design approach utilized in other operational NASA propagation terminals, but has been modified to employ power measurement via a frequency estimation technique and to coherently track and measure the amplitude of the 20/40 $\mathrm{GHz}$ beacon signals. The system consists of a 1.2-m K-band and a 0.6-m Q-band Cassegrain reflector employing synchronous open-loop tracking to track the inclined orbit of the Alphasat satellite. An $8 \mathrm{~Hz}$ sampling rate is implemented to characterize scintillation effects, with a $1-\mathrm{Hz}$ measurement bandwidth dynamic range of $45 \mathrm{~dB}$. A weather station with an optical disdrometer is also installed to characterize rain drop size distribution for correlation with physical based models.
\end{abstract}

\section{TABLE OF CONTENTS}

1. INTRODUCTION .1

2. GRC K/Q-BAND BEACON RECEIVER DESIGN.1

3. FREQUENCY ESTIMATION APPROACH .............3 4. RECEIVER PASSBANd CALIBRATION ...............4 5. DATA ANALYSIS..........................................5 6. REFERENCE ATTENUATION LEVEL..................6 7. CONCLUSIONS ............................................7 REFERENCES....................................................7

\section{INTRODUCTION}

Though the primary focus of NASA's future space communications architecture is in the exploitation of $\mathrm{Ka}$ and optical frequencies, NASA is also investigating the potential use of spectrum in the Ka/Q-bands $(37-42 \mathrm{GHz})$ and V/Wbands (74-84 GHz) as a downlink option in the next generation Space Based Relay (SBR) that is expected to replace the existing Tracking and Data Relay Satellite (TDRS) system in the 2025 timeframe. As such, NASA Glenn Research Center is spearheading a small, collaborative effort to characterize atmospheric propagation effects at these millimeter wave frequencies. A major part of this campaign includes the use of the Alphasat Aldo Paraboni TDP\#5 20/40 GHz beacon in Europe. The primary goal of these measurements is to develop a physical model to improve predictions of atmospheric attenuation within the desired spectrum. Herein, we describe the design and performance of the $20 / 40 \mathrm{GHz}$ beacon receiver, built inhouse by NASA Glenn Research Center, and which has been recording data at the Politecnico di Milano (POLIMI) campus since May 2014. The receiver is based upon the validated FFT digital design approach utilized in other operational GRC propagation terminals [1-2]. The new design incorporates upgrades and modifications to coherently track and measure the amplitude of the 20/40 $\mathrm{GHz}$ beacon signals with improved dynamic range. It is believed that these measurements, combined with thorough characterization of local meteorological conditions, will provide the necessary basis for improved models to assess the performance of communications systems employing frequencies in the Q-band.

\section{GRC K/Q-BAND BEACON RECEIVER DESIGN}

The Alphasat beacon receiver developed at NASA GRC consists of a $1.2 \mathrm{~m} \mathrm{~K}$-band and a $0.6 \mathrm{~m}$ Q-band Cassegrain reflector with equivalent antenna beamwidths of $0.9 \mathrm{deg}$. The receivers employ independent open-loop tracking systems for each antenna to track the inclined orbit of the Alphasat satellite. A block diagram of the front ends and common Intermediate Frequency (IF) downconversion cards (labeled K-DACS and Q-DACS) of the system is provided in Figure 1 and Figure 2, respectively. Primary downconversion to a common IF of $70 \mathrm{MHz}$ is performed within the temperature controlled RF boxes mounted directly behind the antennas. Independent temperature control of the LNA and the plate upon which the RF electronics are mounted maintain a temperature stability of +/- 0.01 deg C. From the RF boxes, the signal is routed to a secondary temperature controlled IF box where final downconversion stages take place to the common $455 \mathrm{kHz}$ IF. Temperature stability of the IF box is maintained to within $+/-0.25 \operatorname{deg} \mathrm{C}$. 


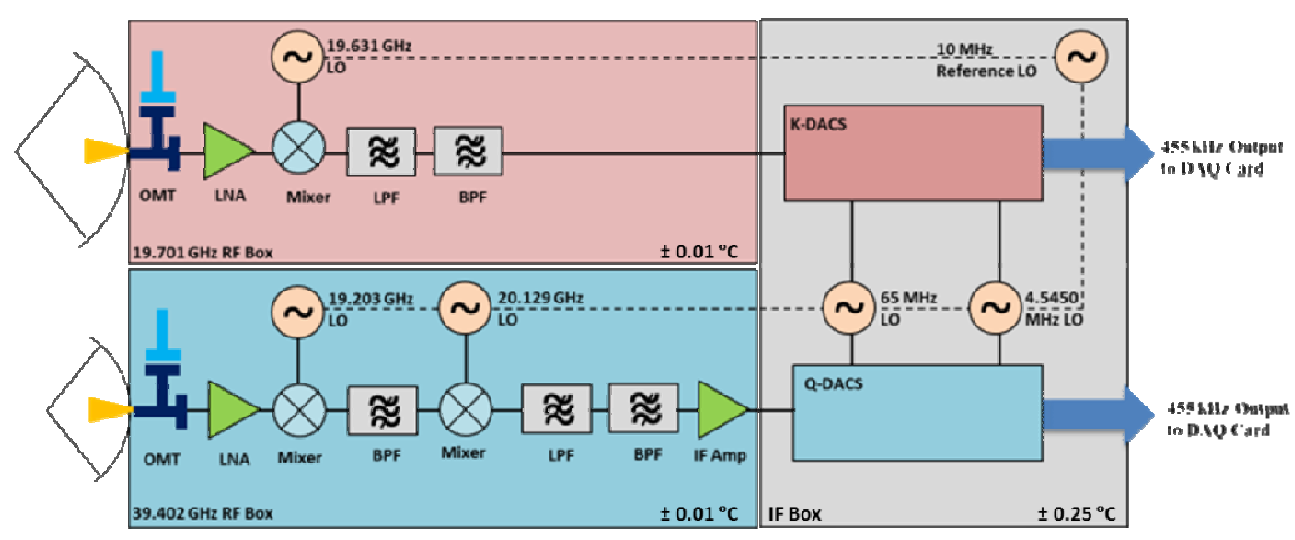

Figure 1 - Block diagram of coherent K/Q-band beacon receiver

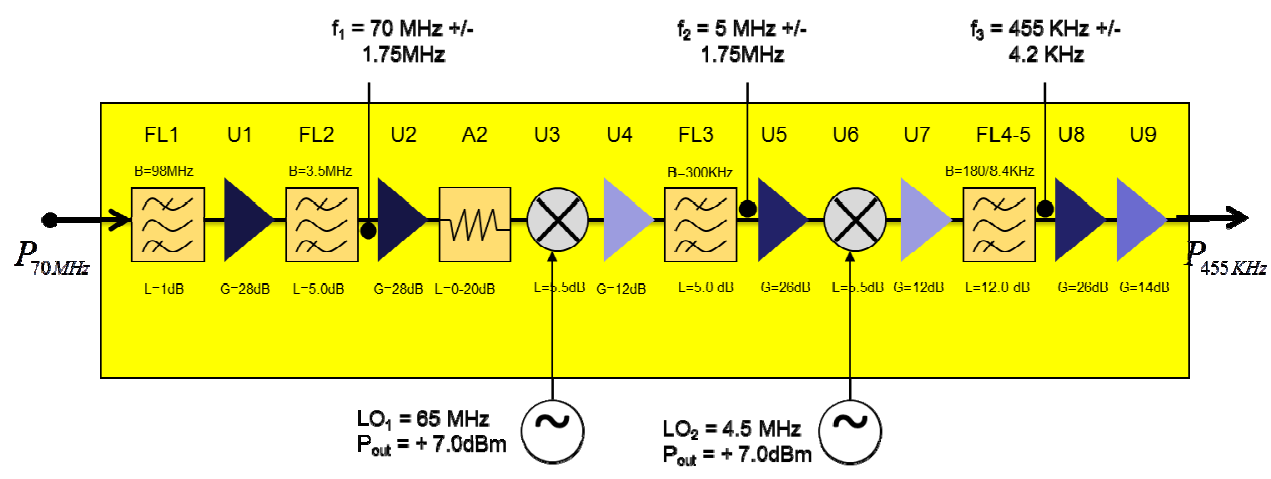

Figure 2 - Block diagram of coherent K/Q-band beacon receiver

The IF Cards (K-DACS/Q-DACS) are integrated circuit boards originally utilized during the ACTS experiment which were subsequently modified to maintain coherent downconversion of the receive beacon signals. The input to these cards necessitate a $70 \mathrm{MHz}$ IF and provide the necessary filtering and final downconversion stages to the ultimate $455 \mathrm{kHz}$ IF. To accomplish this, the $19.701 \mathrm{GHz}$ $\mathrm{K}$-band beacon is first downconverted in the RF box via a single stage to the $70 \mathrm{MHz}$ IF frequency input. Due to the limited availability of components (i.e., local oscillators and filters), the $39.402 \mathrm{GHz}$ signal is downconverted in two stages to obtain the necessary $70 \mathrm{MHz}$ IF signal input to the Q-DACS card. All downconversion stages are referenced to a common ultra-stable $10 \mathrm{MHz}$ reference oscillator housed within the IF box.

From there, the signals are routed via coaxial cable to the control and data acquisition computer. The $455 \mathrm{kHz}$ IF signal is Nyquist sampled by a 12-bit National Instruments 5124 data acquisition (DAQ) card at a sampling frequency $\left(\mathrm{f}_{\mathrm{s}}\right)$ of $1.111 \mathrm{MHz}$. At an $8 \mathrm{~Hz}$ data measurement rate, $2^{17}$ samples are collected for a final fs/ $\mathrm{N}$ resolution of $8.47 \mathrm{~Hz}$. The $8 \mathrm{~Hz}$ power measurements are averaged each second to derive $1 \mathrm{~Hz}$ data and both data sets are recorded to file. Frequency estimation and power measurement of the beacon signal employs a modified Quinn-Fernandes frequency estimation routine, as described in [3] and discussed in more detail in the next section. During deep rain fades, i.e., fades greater than $30 \mathrm{~dB}$, the beacon receiver utilizes the K-band signal for frequency tracking to maintain lock on the Q-band signal. Based on this approach, the measurement range of the Q-band receiver is improved down to a $3 \mathrm{~dB}$ bin signal to noise ratio (SNR) and is capable of reestablishing lock once the signal emerges from the noise. This implementation provides a measurement dynamic range of approximately 38 $\mathrm{dB}$ for the $8 \mathrm{~Hz}$ sampling rate, and $45 \mathrm{~dB}$ for the $1 \mathrm{~Hz}$ samples. Further, by employing a frequency estimation routine, we are also able to derive Doppler parameters for estimates of relative spacecraft position and calibration of gain variation across the passband of our receiver.

The receivers were installed atop the roof of the Dipartimento di Elettronica, Informazione e Bioingegneria (DEIB) building on the POLIMI campus in April 2014 and is shown in the photograph of Figure 3. During initial operations, several anomalies were addressed and operational status was achieved on June 1, 2014. To supplement the beacon receivers, a suite of weather sensors are utilized to obtain surface temperature, pressure, humidity, wind speed/direction, and rain rate. A disdrometer has also been installed at the POLIMI site to obtain drop size distribution, fall velocity, and high resolution rain rate measurements during rain events. 


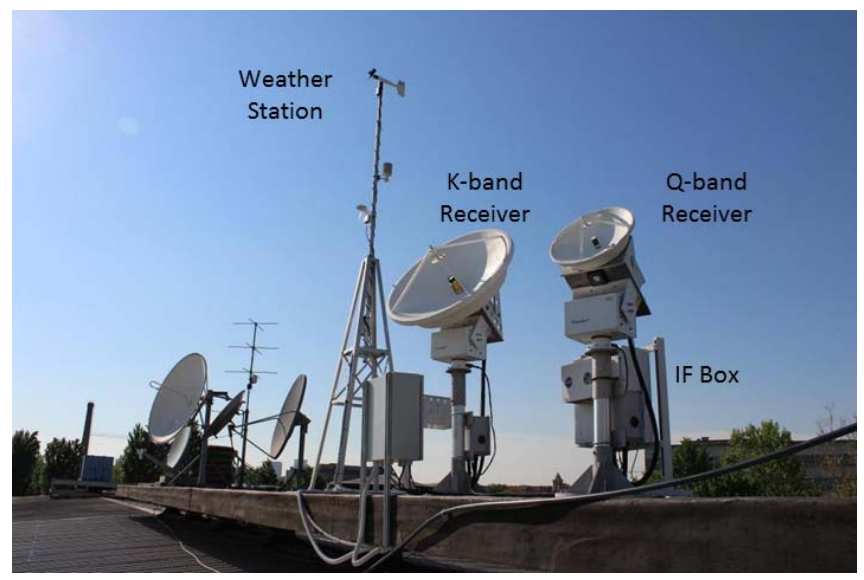

Figure 3 - Photograph of K/Q-band beacon receiver installed at POLIMI campus

\section{FREQUENCY ESTIMATION APPROACH}

For a digital FFT receiver in the absence of a phase-locked loop (PLL), the beacon frequency can drift and must be identified such that a power measurement can be performed. As the frequency drifts from the center of an FFT bin, the signal power will spill over into adjacent bins and, if measuring the peak bin power, will decrease by as much as $3 \mathrm{~dB}$ at the bin edges. This effect can be reduced by summing over multiple FFT bins, but this has the negative effect of reducing the system dynamic range due to the integration of the noise power over a wider bandwidth. One method to alleviate this problem is to employ a frequency estimator to more accurately identify the signal frequency within the peak bin and measure the signal power at baseband.

Figure 4 shows the simulated performance of various frequency estimators as compared to the single bin power estimation technique for an SNR of $-10 \mathrm{~dB}$ [3]. As the simulated frequency is swept between adjacent FFT bins, we observe that any of the identified frequency estimator routines sufficiently track the frequency and provide an accurate power measurement, as compared to the peak FFT approach, where the scalloping effect is readily observable.
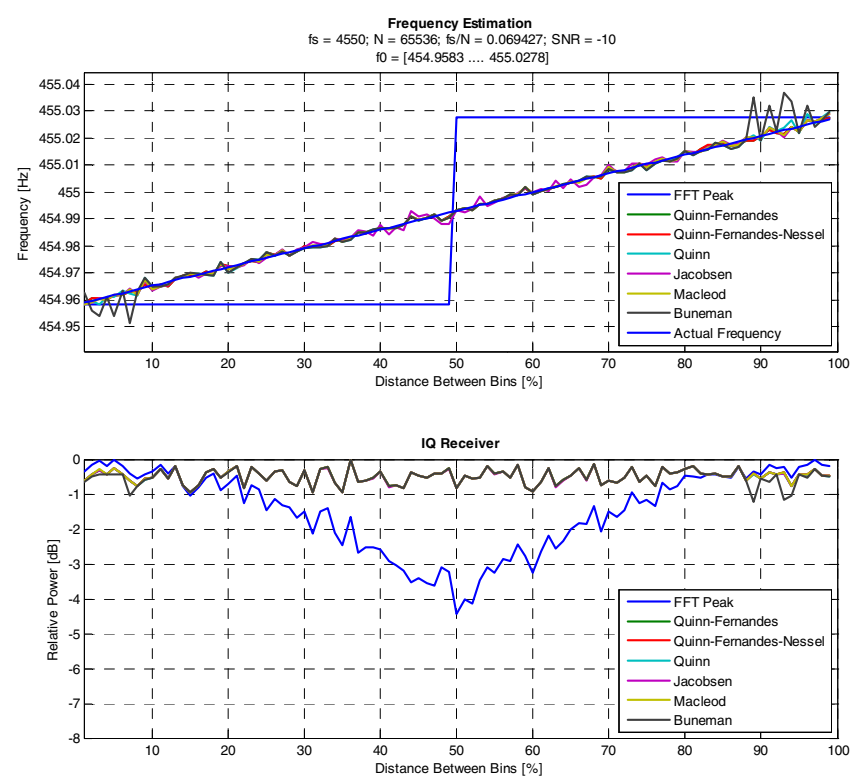

Figure 4 - Frequency estimate of various estimation routines (top) and the corresponding power (bottom) for a simulated -10dB SNR

For our receiver design, we employ the Quinn-Fernandes frequency estimation technique [4]. The Quinn-Fernandes technique attempts to find the local maximum of a smoothed periodogram nearest some initial frequency estimate by optimizing the parameters $\alpha$ and $\beta$ in the filtered spectrum as,

$$
y_{t}-\beta y_{t-1}+y_{t-2}=\varepsilon_{t}-\alpha \varepsilon_{t-1}+\varepsilon_{t-2}
$$

Utilizing this approach, it is possible to derive the frequency estimate within typically 2-3 iterations, provided a good initial estimate is supplied. As we deal with relatively high SNR, this is easily realized. In situations where deep fades occur on the Q-band signal, a modified form of the QuinnFernandes technique is employed in which the initial frequency estimate is supplied by the K-band reference. To determine the Q-band frequency, a band-limited QuinnFernandes algorithm accurately identifies the beacon frequency over a reduced spectral range. In Figure 5, it can be observed that, during a rain event, the frequency estimator is able to track the signal as it approaches the noise floor, and recover it immediately once it returns. 

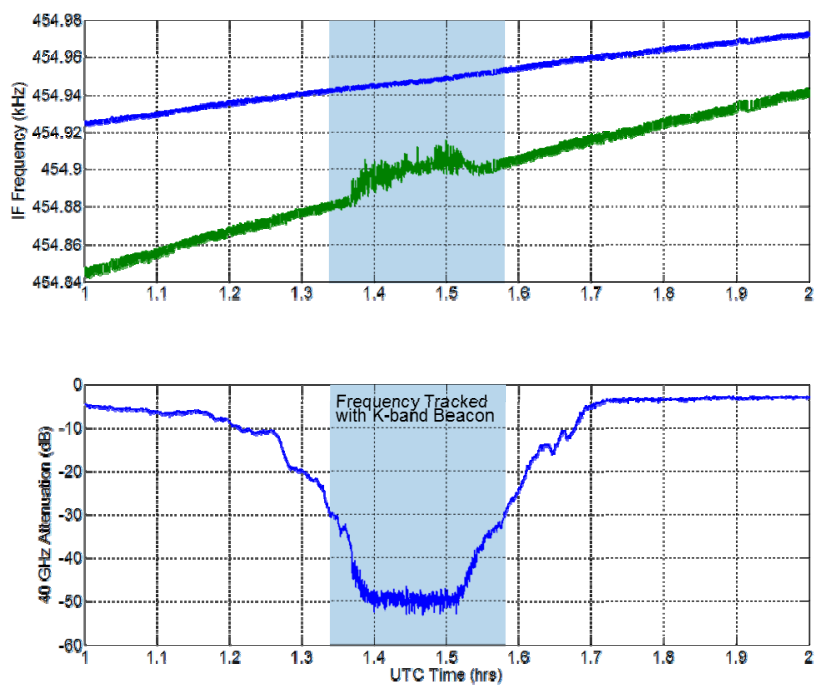

Figure 5 - A deep rain fade event where the Q-band receiver entered frequency track mode (green curve in top plot) utilizing the K-band beacon frequency reference (blue curve in top plot). Note that lock was maintained down to approximately $-40 \mathrm{~dB}$ of attenuation (bottom plot)

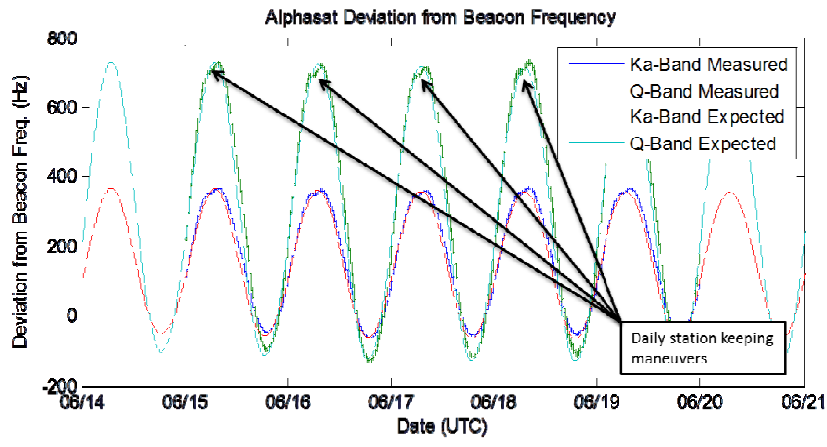

\section{Figure 6 - Comparison of OEM tracking file-derived Doppler shift and measured frequency shift by the receivers}

In addition, the frequency estimation also provides a means to track the relative position of the satellite and differentiate between signal drift and station keeping maneuvers. Plotted in Figure 6 is the deviation from the beacon center frequency induced by Doppler derived from the weekly provided OEM tracking files compared with the frequency estimator. A comparison of these two plots for 1 week show excellent agreement and indicate when station keeping maneuvers are taking place. Also evident throughout the week is a long-term mean frequency drift of approximately $15 \mathrm{~Hz} /$ day (K-band) and $30 \mathrm{~Hz} /$ day (Q-band) which could be a combination of system drift and/or beacon frequency drift.

\section{ReCeiver PASSband CAlibration}

During the system checkout phase, it was observed that a diurnal drift of the signal power was evident in the measured data. Further investigation of this phenomenon resulted in the discovery that the filter passband of the IF cards exhibited some significant variation across the band. In previous campaigns, this effect was not noticeable, but does require calibration for the Alphasat experiment due to the non-trivial frequency variation induced by the Doppler shift of Alphasat's inclined orbit. To calibrate the passband variation of the receivers, the antennas are pointed to zenith on a clear evening and a decimated version of the passband spectrum is recorded to file every second for two hours. The spectrum is subsequently averaged and an 8th order polynomial is fit to the curvature of the passband, as shown in Figure 7. At the completion of each day, the polynomial coefficients are applied to a correction routine to generate calibrated data files. A comparison of the time series before and after a calibration is performed is shown in Figure 8, where it is observed that the calibration routine does sufficiently correct for the gain variation across the band. Characterization of the passband is performed nominally once a month or when major system changes have occurred (i.e., operational temperature changes, power outages) and the coefficients for calibration are updated accordingly.
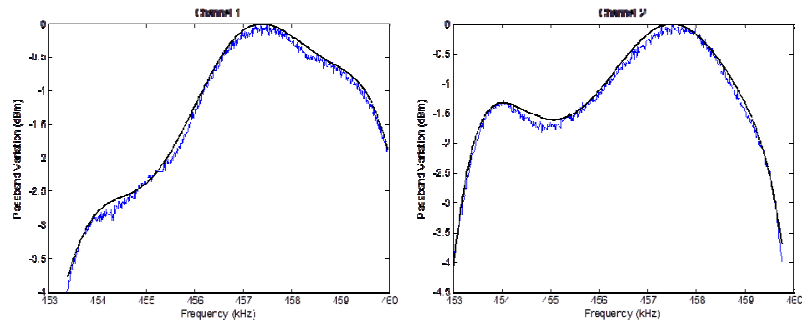

Figure 7 - Measurement and fitting of an $8^{\text {th }}$ order polynomial of the passband spectrum indicating a nontrivial gain variation for (a) the K-DACS and (b) the QDACS
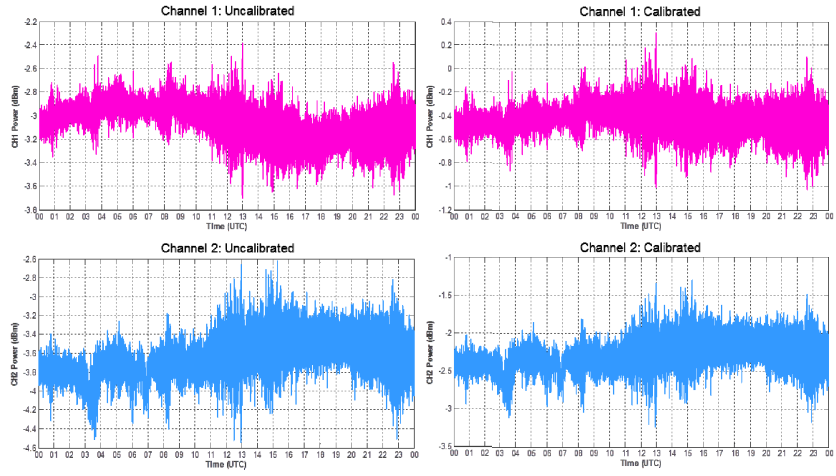

Figure 8 - Example calibration of the gain variation of the passband spectrum for (top) the K-band receiver and (bottom) the $Q$-band receiver 


\section{Data Analysis}

Preliminary analysis of the data has demonstrated excellent performance of the receiver hardware. Analysis of the power spectral density of a representative rain event and scintillation event are shown in Figures 9 and 10, respectively, and agree very well with theory. From the power spectral density plots, we observe that the spectrum of rain and scintillations follow extremely well with the theoretical power law slope of $-20 \mathrm{~dB} / \mathrm{dec}$ and $-80 / 3 \mathrm{~dB} / \mathrm{dec}$, respectively [5]. In Figure 10, we observe for a clear sky day the effects of scintillation are evident at around $0.2 \mathrm{~Hz}$ and saturate at the receiver noise floor of approximately 1 $\mathrm{Hz}$ for the K-band and $2 \mathrm{~Hz}$ for the Q-band. A sample time series for a strong rain event and associated disdrometerrecorded rain rate is shown in Figure 11.
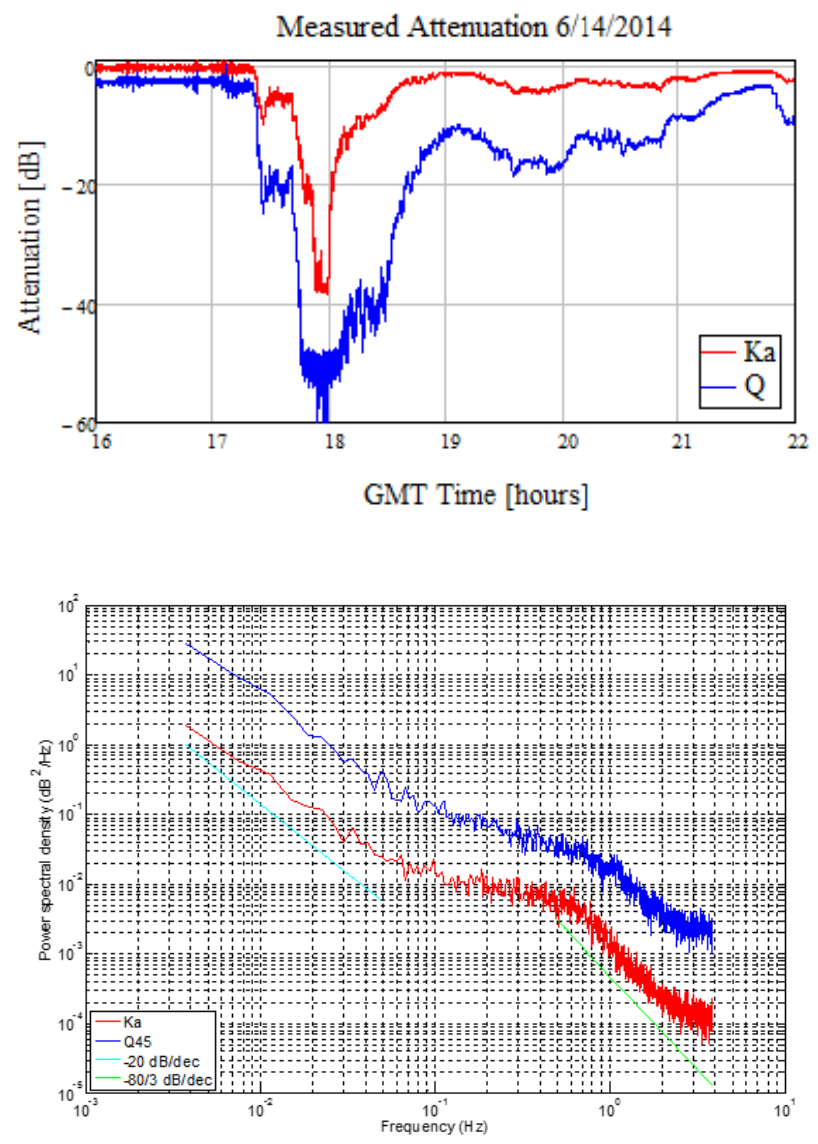

Figure 9 - Rain event time series for 6/14/2014 (left) and the resulting power spectral density plot (right)
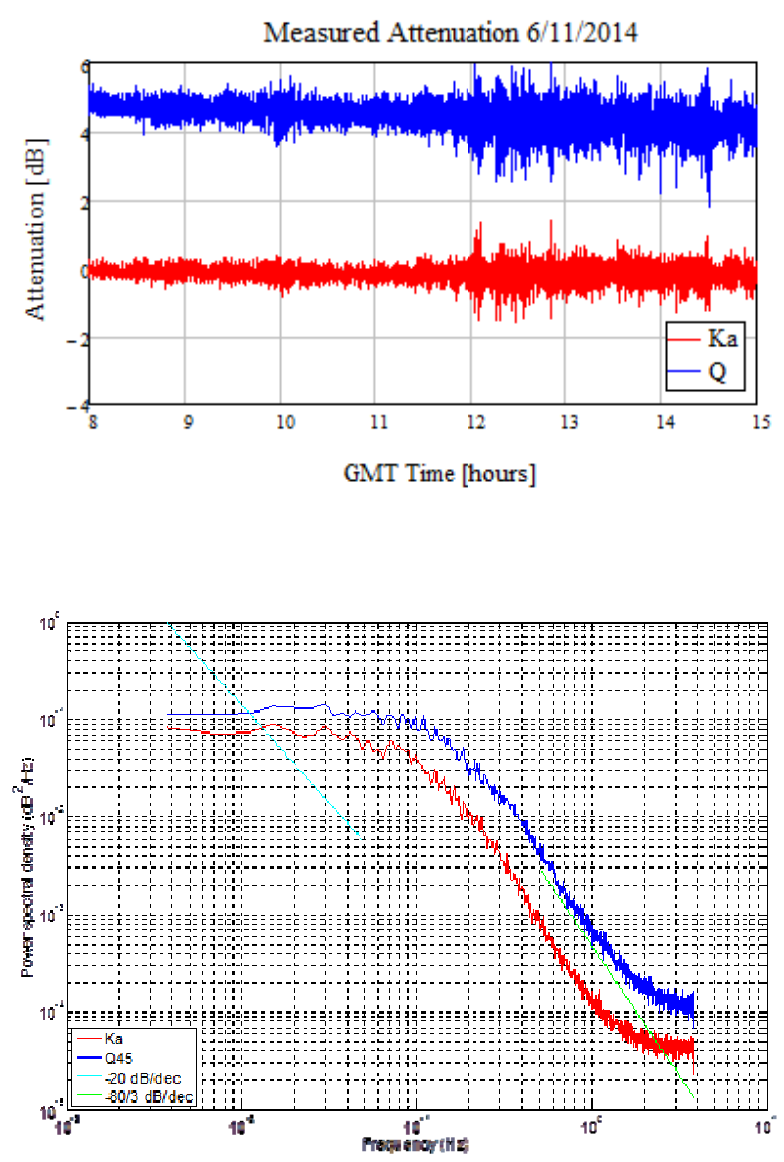

Figure 10 - Scintillation event time series for 6/11/2014 (left) and the resulting power spectral density plot (right)
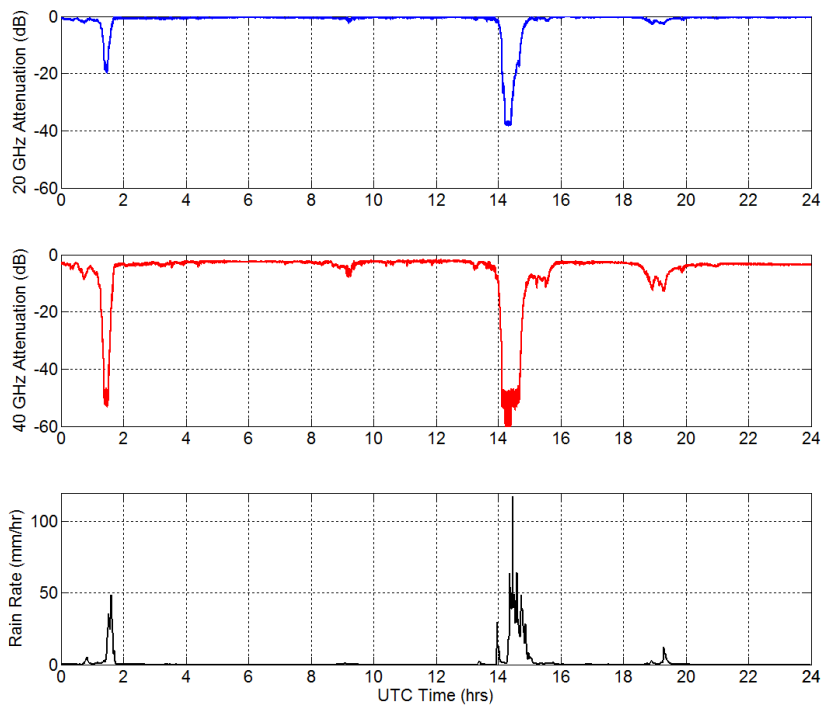

Figure 11 - Time series of a rain event for (top) the Kband receiver, (middle) the Q-band receiver, and (bottom) the disdrometer-recorded rain rate 


\section{REFERENCE ATTENUATION LEVEL}

A key point in obtaining accurate total atmospheric attenuation values from the beacon receivers is the correct identification of the reference attenuation level $A_{\text {ref }}$, which is required to convert the received power levels $(\mathrm{dBm})$ into total attenuation $(\mathrm{dB})$. Radiometers are typically employed to estimate $A_{\text {ref }}$ in non-rainy (i.e. non-scattering) atmospheric conditions starting from the brightness temperature values collected at different frequencies [6].

When no radiometers are available, as it is currently the case for the POLIMI experimental site, radiosonde observations (RAOBS) or operational Numerical Weather Prediction (NWP) products represent a valuable alternative. Figure 12 illustrates the full workflow to achieve power level calibration using the RAOBS data collected at Milano Linate airport (approx. $5 \mathrm{~km}$ away) twice a day (0 and 12 GMT) and the meteorological information produced by the European Centre for Medium-range Weather Forecast $\left(\right.$ ECMWF) on a global $0.125^{\circ} \times 0.125^{\circ}$ latitude $\times$ longitude grid (available at 0, 6, 12 and $18 \mathrm{GMT}$ ).

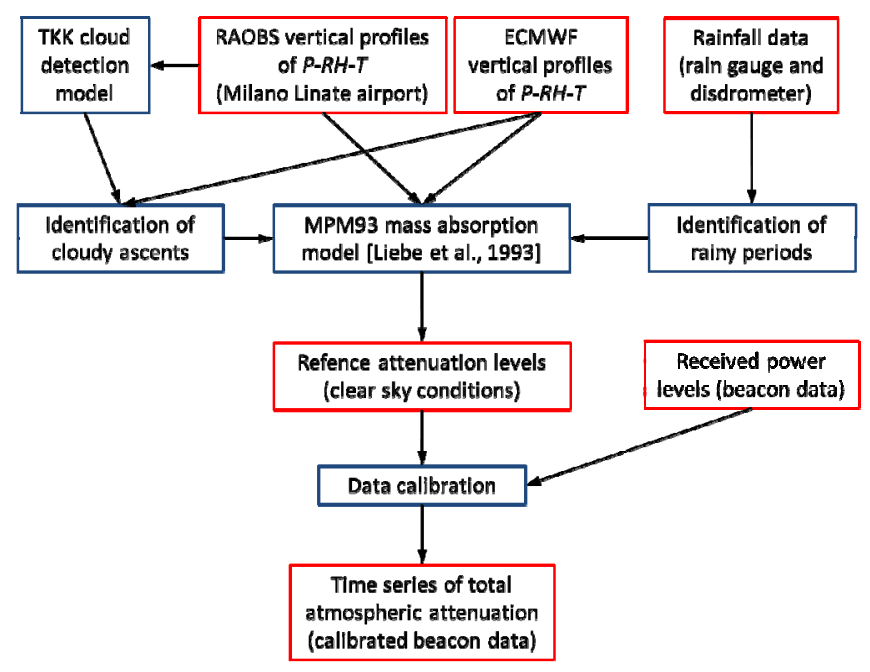

Figure 12 - Detailed workflow for the calibration of beacon power levels using RAOBS and NWP data

Vertical profiles of pressure $(P)$, relative humidity $(R H)$ and temperature $(T)$ are first used as input to the TKK (Teknillinen KorkeaKoulu) cloud detection model [7], to estimate the vertical distribution of the cloud liquid water content, and then turned into path attenuation (due to clouds and gases) using the Liebe's MPM93 mass absorption model [8]. As clarified in Figure 12, we have filtered out all the profiles collected during rain events (exploiting rain gauge and disdrometer data) and, more in general, for which clouds have been identified (either by the TKK model for ROABS of by the ECMWF atmospheric model for NWP profiles). The former type of profiles is very critical, as mass absorption models do not typically take into account the effects of precipitation (while the received beacon signal is extremely sensitive to rain as shown for example in
Figure 13); to a lesser extent, also the presence of suspended liquid water along the profiles reduces the effectiveness of the outline calibration procedure because of the uneven distribution of clouds which might not simultaneously cover both the RAOBS launch site and the experimental site.

As an example, Figure 13 depicts, for all September 2014, the disdrometer-recorded rain rate and $D$, which is defined as:

$$
D=-P_{\text {lev }}-A_{\text {ref }}
$$

where $P_{l e v}$ is the received beacon power level and $A_{\text {ref }}$ is the reference attenuation estimated according to the procedure outlined in Figure 12. Results clearly indicate a fairly good stability of $D$ during clear sky conditions, as it should be the case when the receiver chain is kept stable, both in terms of operating temperature and of settings of the chain elements (e.g. gain of amplifiers). As a final step, we have calculated $D_{m o n}$, the mean monthly value of $D$ (for September 2014, $D_{\text {mon }}=-0.22 \mathrm{~dB}$ and $D_{\text {mon }}=0.77 \mathrm{~dB}$ ) and derived the total atmospheric attenuation $A$ as:

$$
A=-P_{\text {lev }}-D_{\text {mon }}
$$
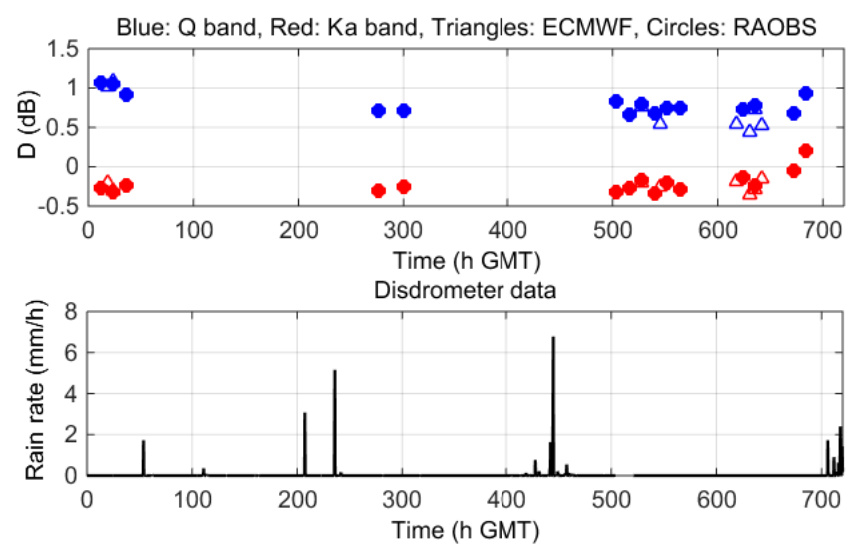

Figure 13 - September 2014: disdrometer-recorded rain rate (bottom) and $D$ values in clear sky conditions (top)

Figure 14 shows an example of the calibrated attenuation data obtained by applying (3) (part of September 2014). 

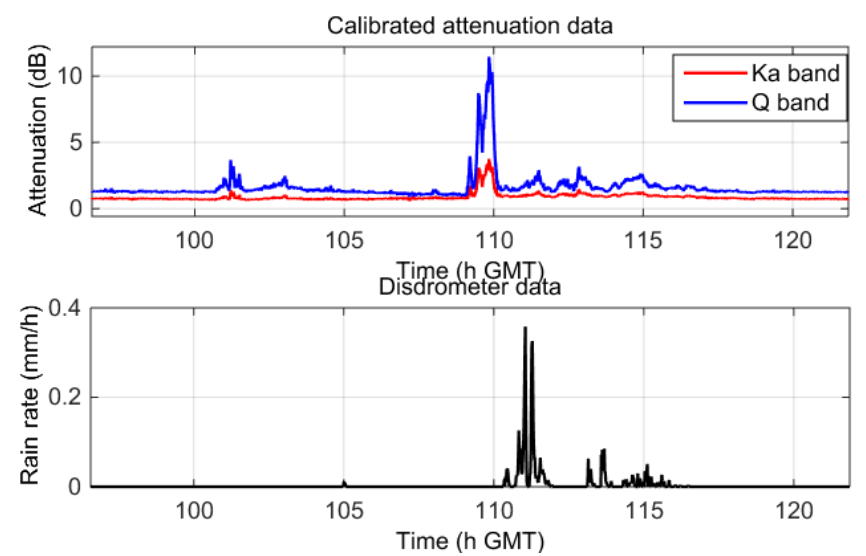

Figure 14 - Example of calibrated attenuation data

\section{CONCLUSIONS}

Herein we present the design and performance of the NASA GRC-developed Alphasat beacon receiver terminals, which has been collecting data at the POLIMI campus since May 2014 and initiated formal operations on June 1, 2014. Barring system issues, the performance of the receiver has exceeded expectations and has been recording reliable data, which validates theoretical models of, for instance, rain and scintillation power spectral density slopes. We employ a modified Quinn-Fernandes frequency estimation routine to derive the frequency and power measurement of the beacon signals, resulting in a demonstrated dynamic range of approximately $38 \mathrm{~dB}$ at the $8-\mathrm{Hz}$ sampling rate, and $45 \mathrm{~dB}$ at the $1-\mathrm{Hz}$ sampling rate. The system calibration routine for passband gain variation has been described which adequately removes this effect. Currently, the zeroreference level calibration is derived from radiosonde measurements collected at Milano Linate Airport (approx. 5 $\mathrm{km}$ away) and from meteorological information provided by the ECMWF. Next steps for this campaign involve the installation of a water vapor radiometer to more directly zero-reference the power measurements. Further, once the ASI site in Spino d'Adda is operational, the opportunity to conduct site diversity measurements at Q-band will be available, as the POLIMI site and the Spino d'Adda site lie approximately $20 \mathrm{~km}$ distance apart.

\section{REFERENCES}

[1] R. Acosta, et. al., "Goldstone Site Test Interferometer", $13^{\text {th }} \mathrm{Ka}$ and Broadband Comm. Conference, Turin, Italy, Sep. 2007.

[2] R. Acosta and J. Nessel, "Data Processing for Atmopheric Phase Interferometers", NASA TM 2009-215599

[3] M. Zemba, J. Morse, J. Nessel, "Frequency Estimator Performance for a Software-Based Beacon Receiver", In Proceedings of the 2014 IEEE Antennas and Propagation Conference, July 6-12, 2014, Memphis, TN.

[4] B. G. Quinn and J. M. Fernandes, "A Fast Technique for the Estimation of Frequency", Biometrika, 78(3) (1991), pp. 489-497.

[5] E. Matricciani, M. Mauri, and C. Riva, "Scintillation and simultaneous rain attenuation at $12.5 \mathrm{GHz}$ to satellite Olympus", Radio Science, Vol. 32, No. 5, pp. 1861-1866, Sep./Oct. 1997.

[6] L. Luini, C. Riva, C. Capsoni, A. Martellucci, "Attenuation in non rainy conditions at millimeter wavelengths: assessment of a procedure", IEEE Transactions on Geoscience and Remote Sensing, pp. 2150-2157, Vol. 45, Issue 7, July 2007.

[7] E. Salonen and S. Uppala, "New prediction method of cloud attenuation", Electron. Lett., vol. 27, no. 12, pp. 1106-1108, Jun. 1991.

[8] H. J. Liebe, G. A. Hufford, M. G. Cotton, "Propagation modeling of moist air and suspended water/ice particles at frequencies below $1000 \mathrm{GHz}$," in Proc. AGARD 52nd Spec. Meeting EM Wave Propag. 
